\title{
A role for central carbon metabolism in mammalian embryonic development?
}

\author{
Marteinn Snaebjornsson ${ }^{1 *}$, Nicole Prior ${ }^{1}$, Vinay Bulusu ${ }^{1}$, Bernd Simon ${ }^{2}$, Teresa Carlomagno ${ }^{2}$, Alexander Aulehla $^{1}$ \\ From Metabolism, Diet and Disease 2014: Cancer and metabolism \\ Washington DC, USA. 28-30 May 2014
}

\section{Background}

Embryonic development is not regulated solely by genetic programs but also by metabolic state and environmental cues, which can impact on gene function. Metabolism can influence gene function both canonically (providing substrates for posttranslational modifications etc.) and non-canonically for example via moonlighting enzymes. Moonlighting enzymes have functions in addition to their known catalytic one. They are widespread among enzymes involved in sugar metabolism. For example, 7 out of the 10 enzymes in glycolysis have confirmed roles as moonlighting enzymes [1]. We study the role of glycolysis during mouse embryonic development. To this end, we perturb carbon metabolism in presomitic mesoderm (PSM) explants in culture. Several attributes of the PSM make it an attractive choice for our studies, first of all it can be cultured in vitro for extended time periods and displays normal development during culture. This allows us to analyze the effect of our perturbations in a developmental context at the morphological and molecular level.

\section{Materials and methods}

We culture PSM explants in a defined medium in which we can control precisely the nutrient source for the explants, for example replace glucose with a glycolytic intermediate. To analyze the effects of our perturbations we use a transgenic reporter line and real time imaging. We measure lactate secretion and use C13 isotopic labeling to estimate whether and how much glycolytic intermediates are taken up by explants. To search for indications of moonlighting activity of glycolytic enzymes we analyze their localization using subcellular fractionation.

\section{Results}

We find that culture in medium containing F1.6bP more than any other glycoytic metabolite strongly affects PSM development. Additionally we find that a subset of glycolytic enzymes show an unexpected subcellular localization in mouse embryos. Interestingly this localization is altered in the presence of glycolytic intermediates in the culture medium.

\section{Conclusions}

We have evidence suggesting a non-canonical role for glycolysis during embryonic development. Our finding that the localization of glycolytic enzymes can be altered by the presence of metabolites indicates a possible link between metabolic flux and other cellular processes.

\section{Authors' details}

'Developmental Biology unit, EMBL, Heidelberg, Germany. ${ }^{2}$ Structural and Computational Biology unit, EMBL, Heidelberg, Germany.

Published: 28 May 2014

\section{Reference}

1. Sriram G, et al: Single-gene disorders: what role could moonlighting enzymes play? Am J Hum Genet 2005, 76:911-924.

\section{doi:10.1186/2049-3002-2-S1-P69}

Cite this article as: Snaebjornsson et al: A role for central carbon metabolism in mammalian embryonic development? Cancer \& Metabolism 2014 2(Suppl 1):P69.

'Developmental Biology unit, EMBL, Heidelberg, Germany

Full list of author information is available at the end of the article 